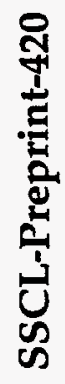

SSCL-Preprint-420

May 1993

Distribution Category: 400

D. Veal

\title{
Industrial Metrology as Applied to Large Physics
} Experiments

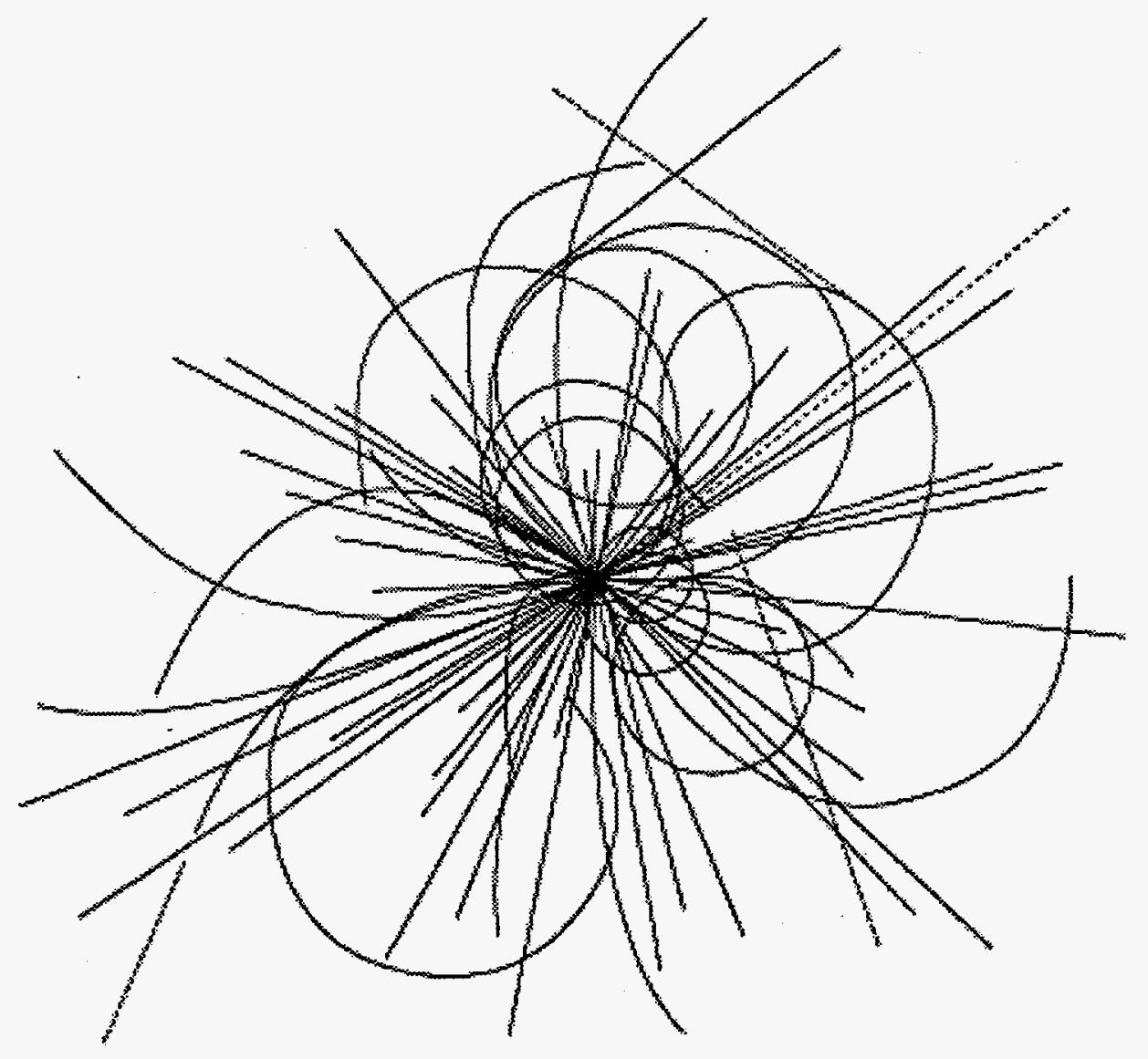

APPROVED FOR RELEASE OR PUBLICATION - O.R. PATENT GROUP BY... \& ....... DATE. $1 / 3 / 25$.

\section{Superconducting Super Collider Laboratory}




\section{Disclaimer Notice}

This report was prepared as an account of work sponsored by an agency of the United States Govemment. Neither the United States Government or any agency thereof, nor any of their employees. makes any warranty, express or implied, or assumes any legal liability or responsibility for the accuracy, completeness, or usefulness of any information, apparatus, product, of process disclosed, or represents that its use would not infringe privately owned rights. Reference herein to any specific commercial product, process, or service by trade name, trademark, manufacturer, or otherwise, does not necessarily constitute or imply its endorsement, recommendation, or favoring by the United States Government or any agency thereof. The views and opinions of authors expressed herein do not necessarily state or reflect those of the United States Government or any agency thereof.

Superconducting Super Collider Laboratory is an equal opportunity employer. 


\section{DISCLAIMER}

Portions of this document may be illegible in electronic image products. Images are produced from the best available original document. 
SSCL-Preprint-420

\title{
Industrial Metrology as Applied to Large Physics Experiments
}

D. Veal

\author{
Superconducting Super Collider Laboratory* \\ 2550 Beckleymeade Ave. \\ Dallas, TX 75237
}

May 1993

\section{DISCLAIMER}

\begin{abstract}
This report was prepared as an account of work sponsored by an agency of the United States Government. Neither the United States Government nor any agency thereof, nor any of their employees, makes any warranty, express or implied, or assumes any legal liability or responsibility for the accuracy, completeness, or usefulness of any information, apparatus, product, or process disclosed, or represents that its use would not infringe privately owned rights. Reference herein to any specific commercial product, process, or service by trade name, trademark, manufacturer, or otherwise does not necessarily constitute or imply its endorsement, recommendation, or favoring by the United States Government or any agency thereof. The views and opinions of authors expressed herein do not necessarily state or reflect those of the United States Government or any agency thereof.
\end{abstract}

* Operated by the Universities Research Association, Inc., for the U.S. Department of Energy under Contract No. DE-AC35-89ER40486. 


\section{INDUSTRIAL METROLOGY AS APPLIED TO LARGE PHYSICS EXPERIMENTS}

David Veal, MSc Eng, Section Head Detector Alignment, Superconducting Super Collider Laboratory, USA

\section{ABSTRACT}

A physics experiment is a large complex 3-D object (typ. $1200 \mathrm{~m}^{3}, 35000$ tonnes), with sub-millimetric alignment requirements. Two generic survey alignment tasks can be identified; first, an iterative positioning of the apparatus subsystems in space and, second, a quantification of as-built parameters.

The most convenient measurement technique is industrial triangulation but the complexity of the measured object and measurement environment constraints frequently requires a more sophisticated approach. To enlarge the "survey alignment toolbox" measurement techniques commonly associated with other disciplines such as geodesy, applied geodesy for accelerator alignment, and mechanical engineering are also used. Disparate observables require a heavy reliance on least squares programs for campaign pre-analysis and calculation.

This paper will offer an introduction to the alignment of physics experiments and will identify trends for the next generation of SSC experiments.

\section{INTRODUCTION}

The Superconducting Super Collider Laboratory has been established to design, build, maintain, and operate the Superconducting Super Collider (SSC), a high energy subatomic particle accelerator that will be used in basic research to learn more about the fundamental nature of matter and energy. When completed in 1999, the Super Collider will be the most powerful sub-atomic particle accelerator in the world. In the collider two separate beams of protons will travel in opposite directions at near light speed. At specific points on the east and west sides of the rings, the beams will be brought into interaction. The particles engendered by the collision will be recorded in the physics experiment that surrounds the interaction point. The analysis of the data will provide valuable information on the basic building blocks and forces of nature.

A collider experiment at the SSC is a very sophisticated measurement apparatus of great size, weight and complexity housed in an underground cavern of commanding proportions (typ. $100 \mathrm{~m} \times 30 \mathrm{~m} \times 30 \mathrm{~m}$ ). The experiment is subdivided into many parts, each subsystem measuring a particular aspect of the interaction.

Geodetic engineers are involved in many survey alignment tasks at the larger particle physics laboratories, in particular in the alignment of the accelerators and the detectors. This paper will concentrate on survey alignment of physics detectors.

\section{TASKS}

Survey alignment because of its dual role in the alignment of the machine and the experiment provides the best estimate of the trajectory of the particle beam until commissioning of the accelerator. The tasks of iterative positioning and description are performed with respect to this expected interaction point and trajectory of the particle beam. Iterative Positioning (Placement)

The task involves the iterative movement of subsystem elements to their theoretical position. Failure in this task will introduce biases into the physics data because the decision to accept or reject an event is made using hardwired logic, which relies on the realization of theoretical form. This initial alignment task also assures that positioning errors on the experiment do not prevent the accelerator from reaching design parameters. This is of particular importance for the experimental magnet and beam tube.

Description

This task involves the documentation of the as built parameters of the experimental apparatus. This form is reconstructed from a variety of sources including the matching of particle tracks, survey alignment measurements and internal monitoring systems. 


\section{Other Tasks}

Survey alignment provides technical support to the collaboration during assembly and installation of the experimental apparatus. This includes quality control measurements on manufactured parts, setting out of jigging, documentation of form during assembly and the prealignment of interface components (rails, rollers, etc.).

\section{CHALLENGES \\ Experiment Size and Structure}

The sheer size of the experiment (see Fig. 1) is a major challenge to survey alignment. The aim is to maximize optical coverage of the detector.

A physics experiment is typically arranged in concentric layers, each subdivision measuring different aspects of the interaction. The detector as a whole is designed to be as hermetic as possible so that all products of the interaction are recorded. Once the experiment is completely installed and assembled optical access to the inner layers is very limited.

\section{Experiment Services}

Experiment services for readout and control of the detector have to be channeled out of the detector. This mass of pipes and cables is of immediate concern to survey alignment because if uncontrolled it will restrict measurement geometry and obscure fiducial marks.

Alignment Requirements

Typically placement requirements are of the order of millimeters and have to be maintained in operating conditions. In order to satisfy such requirements finite element analysis results are taken into account, to assure that measurements made on a partly complete structure are compensated for future evolution of the shape due to progressive loading, heat load and magnetic fields.

Description requirements are generally sub-millimetric and in the case of the innermost elements tens of microns are usual. It is essential for the success of the experiment that as-built parameters be maintained to this level throughout the operation of the detector. Sirvey alignmint can only document form before start up. The larger experiments being built for both the SSC and the Large Hadron Collider (LHC) will rely very heavily on internal monitoring systems to document the form of the ensemble

\section{Cavern Environment}

The experimental cavern is very expensive real estate and as such, space is at a premium. It is a multi-user environment where survey alignment is only one of the many techrical users with space requirements. It is not always a laboratory environment, in particular during the early stages of installation, it has more in common with a shipyard.

Multi-user Environment

An experimental collaboration is a multi-national community numbering several hundreds of people from several 10's of institutions. The assembly and installation call upon the skilis of people from many areas of technical expertise. Responsibility for installation and assembly rests at sub-system level with overall control and integration issues being handled by the experiment project manager's office. In order to be successful survey alignment must get involved at many levels of this organization.

\section{STRATEGIES FOR THE ALIGNMENT OF LARGE PHYSICS EXPERIMENTS}

The methods described in this section, were used in the alignment of the Large Electron Positron Collider (LEP) at the Center for Nuclear Research in Geneva, Switzerland. The LEP is the largest particle accelerator in the world today. The responsibility for the alignment work of the four LEP experiments was shared by the author and C.Lasseur. This work is documented elsewhere in more detail.(e.g. Lasseur 1990)

Mechanical Design

The short sight lengths and stringent alignment requirements that typify survey alignment for physics experiments oblige the use of forced centering systems. The many varied tasks and particular environment, often require the development of specialized instrumentation and jigging. Mechanical design is a very important tool in the survey alignment of physics detectors. 


\section{Cavern Network}

A 3-D coordinate system is established oriented with respect to the particle beam and with the origin at the interaction point. The spatial location of this reference frame must be maintained throughout the installation of the experiment. The materialization of this reference frame, the cavern geodetic network, will be designed to maintain good internal consistency of relationship and to give a general coverage of the installation and assembly zones in the cavern. Particular placements will be chosen to facilitate certain alignment tasks.

The physical constraints of the cavern, in particular, the position of walkways and the multi-user environment, severely limit possible locations for network points. The use of points along walkways facilitates description of the object, but they must not be an obstruction in an emergency. A design that was successfully used at the LEP was a folding survey bracket attached to the cavern wall at a convenient height with respect to the walkway. These survey brackets were equipped with a forced centering system to assure the repeatability of stationing to machining tolerances and could be folded back against the wall when not in use to liberate the passage along the walkway.

\section{Large Tool Box}

The number and variety of survey alignment tasks and the difficult measurement environment on physics experiments require great adaptability of method to application.

- Industrial metrology

The workhorse technique is industrial triangulation. The principal advantages of this method are its accuracy, it does not require contact with the measured object, and its flexibility of usage. Industrial triangulation is used both on- and off-line with the adjustment by bundle ray or classical geodetic observation relationships. The limitation of commercial software has required the development of in-lab software.

- Mechanical measurement devices

Mechanical distance measurement devices, such as calipers can be very useful both in an integrated or a stand-alone measurement solution.

- Applied Geodesy for Accelerators

The DISTINVAR, a distance measuring device using invar wire as a mechanical standard, and Nylon Offset device, a means of measuring offsets from a stretched wire reference, are used for particular measurement applications and as a QC device on optical measurement techniques (Mayoud et al. 1990).

- Commercial

The ambient air conditions in the experimental caverns are reasonably well controlled and it is common to use mechanical forced centering. In such situations, calibration of commercially available distance meters can improve measurement accuracy. This can be an in-situ calibration, using high redundancy to determine additive constants or a periodic calibration to a standard on an interferometric bench. In the latter case, the density of comparison can be such as to allow curve fitting and the modeling of cyclic error if this is appropriate. The Kern ME5000 and the Wild DI2000 are commercial instruments which with careful calibration have yielded better than specified results.

\section{Integrated Approach}

The reconciliation of such disparate observables is made using rigorous least squares adjustment programs. Such programs are developed in lab to cover all aspects of the alignment of the accelerators and experiments (Iliffe 1987). The restriction of the measurement environment means that it is not always possible to employ simple measurement schemes. In such cases the introduction of different observables can significantly strengthen the solution. Measurement campaign planning and optimization in such hybrid measurement schemes cannot be handled intuitively. Rigorous pre-analysis in such cases is essential.

Fiducialization

The physics fiducial marks are often inaccessible and unsuitable for direct measurement. The definition of suitable survey reference mark is of crucial importance because a failure of relationship at this level can never be recovered. To be most effective, fiducials must be defined at an early stage during the manufacture of the component; however at this stage, installation sequences and, consequently, survey alignment tasking is unclear. To maintain flexibility of measurement method, a precisely machined hole was used as a fiducial so that any targeting with 
an appropriately machined male piece could be used. With a sliding fit tolerance, this type of fiducial is suitable for $0.1 \mathrm{~mm}$ measurement.

Prior Knowledge

Reference has already been made to the hermetic structure of the experimental apparatus. In such cases a solution for survey alignment is to deduce the inside from the outside. This solution requires a complete measurement of the object prior to its insertion into the experiment, the old coordinates can then be mapped onto the new for the common points using Helmet transformation, this deduced relationship can then be applied to find new coordinates for the hidden points. Another possibility is to use parameters deduced from previous calibration measurements to strengthen the solution at a later date when the geometry of the measurement is degraded, for example distances between reference points on a plate. Such 'black box' approaches must be used with extreme caution, as they rely on mechanical invariance and experienced judgment.

\section{STRATEGIES FOR THE ALIGNMENT OF VERY LARGE PHYSICS EXPERIMENTS}

The experiments for the SSC will be 2 to 3 times the size of the LEP detectors. Requirements on the other hand are often more stringent and potential movements due to the mass of the experiments amplified.

Advances in Portable Measurement Technology

Two major tools of potential use in the alignment of very large physics experiments have been identified to date.

- The laser tracker's principal assets for the survey alignment of physics experiments are the interferometric distance which allows a measurement to $1 \times 10^{6}$ and its speed of data capture. This latter capability will significantly facilitate the QC of large mechanical pieces. The use of such instrumentation will necessitate a change in fiducial design to be compatible with the 1.5 in. retrosphere target and the accuracy of this instrument.

- Industrial photogrammetry

Industrial photogrammetry is notable because the speed of data capture is independent of the number of measured points and because the instrument station does not have to be stable in the traditional sense. Photographs may be taken from a basket on a crane!

Internal Monitoring Systems

With the very large physics experiments, internal monitoring systems appear to be coming of age. The design and implementation of these systems are the responsibility of the experimental collaboration. Survey alignment provides technical expertise for the design and preanalysis calculation and will be heavily involved in both the prototyping and the installation of these systems.

- Linear geometry

Typical instrumentation includes means of measuring deviations from a straight line, hydrostatic levels, and tiltmeters. The straight line reference can be optical or a tensioned wire. This instrumentation is relatively compact, precise, and operated remotely. It can be used to penetrate the experimental apparatus and be arranged in either a projective geometry, i.e., parallel to the particle tracks or in network. The level of redundancy in these networks is considerably reduced by space constraints; consequently, they are vulnerable to error buildup and sensor failure.

- Spatial geometry

A complementary approach is to use trilateration to describe the external form of the experimental apparatus. The measurement process must be totally automatic, including the pointing of the instrument and requires distance meters capable of measuring to better than $0.1 \mathrm{~mm}$ in a hostile environment including radiation and magnetic field.

\section{Network Densification}

For effective and accurate use of angle measurement to industrial metrology type requirements, the sight length must be minimized. The size of the experimental caverns for the SSC experiments means that a network of stations along the cavern walls is not sufficient. The basic network will be supplemented by stations attached to the more solid parts of the experimental apparatus. The coordinates of these points will be determined from the wall points using high precision distance meters such as the Kern ME5000. 
Great care must be taken when using a dense network not to over constrain the measurements on the object. Movements of the structure supporting the station will introduce inconsistencies into the network. The restrictions of the cavern environment will often mean that the known relationship between points in the cavern network is essential to achieve the required precision, but whenever possible the description of the object should be in a purely local reference frame, i.e., bundle type adjustment and then the mean transformation deduced from observations to the network be applied to the object as a whole.

Fixed Detector Axis Throughout the Time of Installation

The last generation physics experiments were built in a garage position and were then rolled onto the beam line using rails and rollers systems. This meant that final alignment with respect to the particle beam could be completed just before start up and with the final geometry of the interaction region magnets already established. For at least one of the experiments at the SSC, this will not be the case. This is not a major problem in the vertical direction because the experimental apparatus will be mounted on jacks. However, in the horizontal plane the implication for this is that the reference axis of the experiment has to be established at the beginning of installation and maintained until start up, a period of more than three years.

The internal consistency of the cavern geodetic network will be maintained primarily using Kern ME5000. The frequency of re-measurement will be a function of the rate of deterioration of the relationship and the installation requirements. As the installation of the apparatus progresses, many sight lines will be obstructed by the experiment itself, in particular the relationship between the two ends of the detector will be reduced to a non-braced quadrilateral. This relationship is crucial because it will also be required to align the interaction region magnets. The cavern environment at the time of the alignment of the interaction regions magnets is illustrated by Fig. 2 .

In order to assure these crucial relationships and fix the spatial position of the particle orbit from a early stage, the experimental caverns will be equipped with vertical and horizontal benchmarks. The vertical benchmark will either be of standard NGS design or an extensometer. The horizontal bench mark will be an inverted pendulum. The position of these benchmarks will be included in the determination of the accelerator geodetic reference network. This determination will occur immediately after beneficial occupancy of the experimental cavern. The benchmarks will consequently be used to relocate in space successive epochs of the cavern reference network and to close out the final alignment of the interaction region magnets.

\section{REFERENCES}

Lasseur, C. (1990). "Geodetic Metrology for Large Experiments", Proceedings of the Second International workshop on accelerator Alignment, DESY Hamburg, Federal Republic of Germany, Sept 10-12, pp 345-357.

Mayoud, M., Menant., E., and Quesnel, J.P (1990). "Technological Evolution of Measurement Tools Dilemmas, Illusions and Realities", Proceedings of the Second International workshop on accelerator Alignment. DESY, Hamburg, Federal Republic of Germany, Sept 10-12, pp 229-236.

Iliffe, J (1987). "Three-Dimensional Adjustments in a Local Reference System", Proceedings of the CAS : Applied Geodesy for Particle Accelerators. CERN, Geneva, Switzerland April 14-18, pp 307-327. 


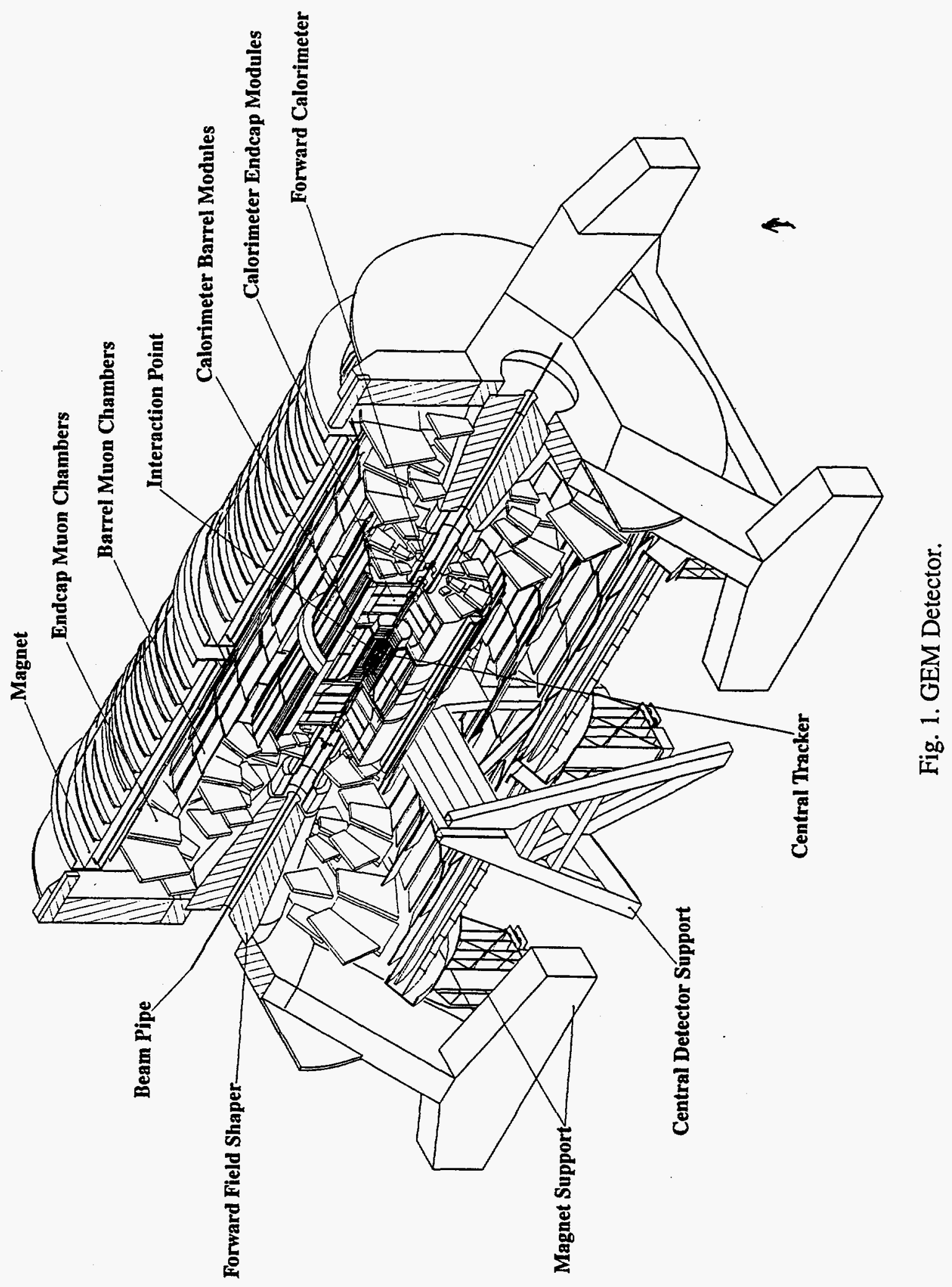




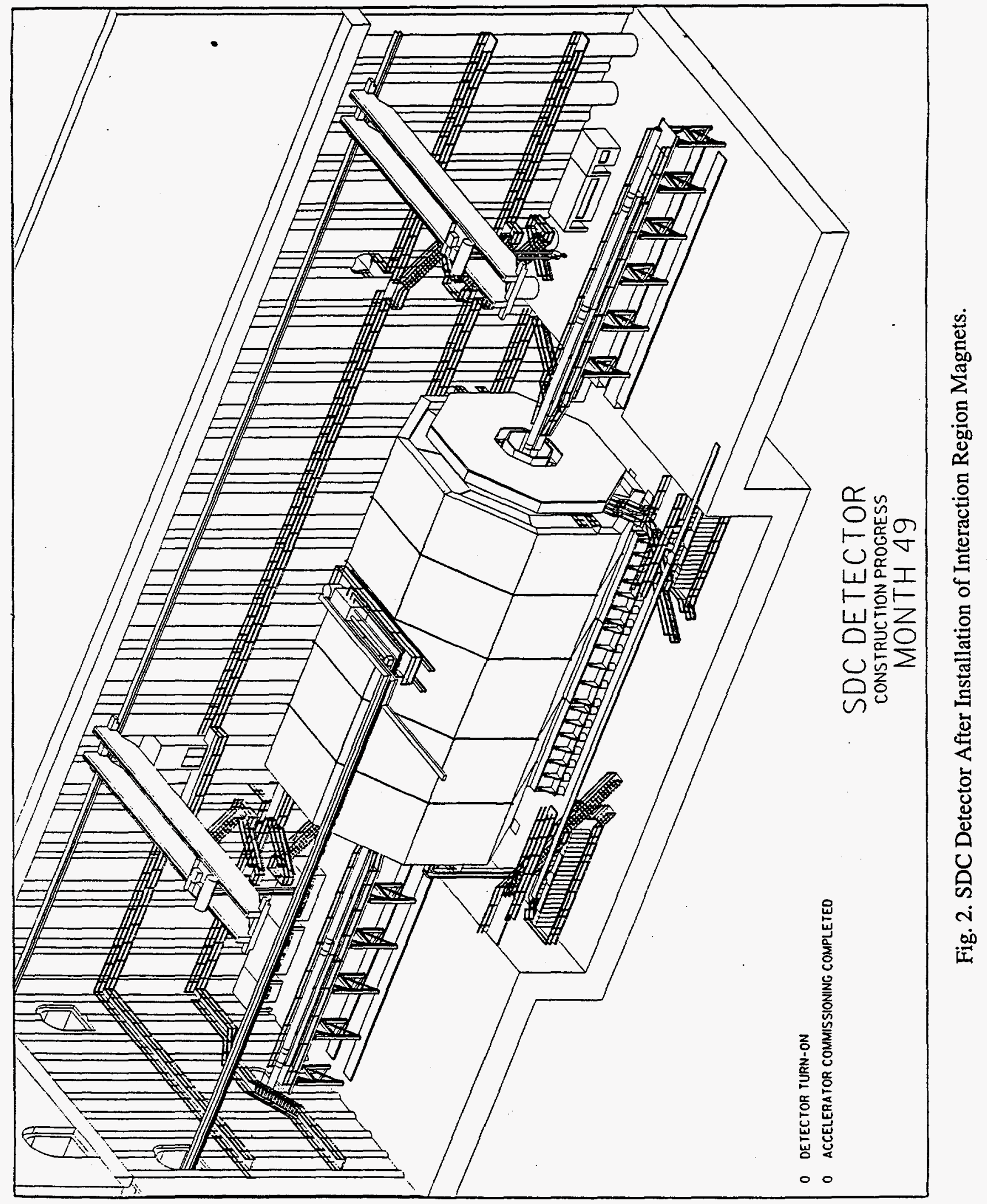

